\title{
Design and Implementation of GPS Based Medical Services using Drone
}

\author{
Rahul S R,V. Arun, SVS. Prasad
}

\begin{abstract}
This paper is aimed to provide medical assistance to people through the delivery of medical supplies by unmanned drones. The use of unmanned drones' benefits people in distant areas around the world. The paper gives attentionto the design of a biocompatible payload and a drone to accomplish medical supply delivery. Design of the drone healthcare delivery network isfacilitatingstructured and low-cost delivery of healthcare to save lives.

Dronesare capable of sustainable flight, which need not human presence on board, and have enough control for performingnecessary functions. This paper utilizes a range of hardware components and software platforms that were integrated into the overall design of the medical drone. Hardware components includes IMU enabled GPS, ArduCopter 2.6 Flight Controller, Data Transmission and Receiving module, Electronic Speed Control Circuits, DC Motors, Propellers, LiPo battery, Servo Connectors. GPS is used for navigation in betweenground stations that are automated, to deliver necessary medications in locations that lack enough roads. Unmanned aerial vehicle is remotely or automatically controlled. Software platform used is ArduPilot Mission Planner and mobile phone or tabletapps can be used to track and navigate. Network is managed by drone operating system to monitor weather data from every ground stationand it optimizes the drone routes in this proposed approach we are designing a paper which is effective in many applications like transporting blood and small emergency medicines such as first-aid kits. Unmanned Aerial Vehicles can be used in future to provide medical help in field of surveillance and at the time of earthquake, drones help rescuers to locate survivors. The military in combat can use drones, and it can also be used for humanitarian aid.
\end{abstract}

\section{INDIAN AEROSPACE INDUSTRY}

The Indian Aerospace Industry is witnessing a remarkable development. Hindustan Aeronautics Limited (HAL) that is totally taken over by the Government of Indiaholds the place of thetopmost aviation organization in the nation. It has assumed a good job in Defense flight through plan, assembling and redesign of warriors, coaches, helicopters, transport air ship, and aeronautics. HAL presently stands 34th in the rank list of world's best hundred barrier organization.

HAL is a good accomplice for the Space projects of Indian Space Research Organization (ISRO) and creates structures and gatherings for dispatching satellites at the committed Aerospace Division in Bangalore. The common flying division in India is developing quickly. It has recorded yearly development of over $41 \%$ in traveler traffic amid over the most recent two years. Truth be told, it has contributed fundamentally to the development of global common flight area. The fast development of common flight has put outrageous weight on the current common aeronautics' foundation. Accordingly, the push is presently on modernization of air terminals, correspondences, route and observation frameworks

Revised Manuscript Received on 14 August, 2019.

Rahul S R, PG Student, Department of ECE,MLR Institute of Technology, Hyderabad, Telangana, India.

V. Arun, Associate Professor, Department of ECE, MLR Institute of Technology, Hyderabad, Telangana, India.

SVS. Prasad, Professor, Department of ECE, MLR Institute of Technology, Hyderabad, Telangana, India for air traffic the board, radars and offices for Maintenance Repair and Overhaul of flying machine and subsystems.

There are consequently gigantic potential and enormous open doors for cooperation and making of joint endeavors in the aviation area in India for setting up Maintenance Repair Overhaul (MRO) offices for common and military flying machine, redesign and support of air motors and creation of flight, parts and embellishments both in the common and military aeronautics divisions. Major worldwide aeronautics industry is as of now looking at the nearby market in India and exploring for reappropriating aviation and guard items as India is quick developing as a middle for building and configuration administrations.

\section{BLOCKDIAGRAM}

Ardupilot Mega (APM), its'san open source unmanned elevated vehicle (UAV) stage, self-ruling multi-copters, fixed wing flying machine, customary helicopters, ground wanderers and reception apparatus checkers. It is an honor winning stage that alsotook over the winning award in 2012 and 2014 UAV Outback Challengerivalries.It began in 2007 with individuals from the DIY Drones people group, rendition bolstered just fixed wing airplane that depended on a thermopile sensor thatisdependentfor deciding the area of the skyline with respect to flying machine by estimating the distinction between temperature of the sky and ground.

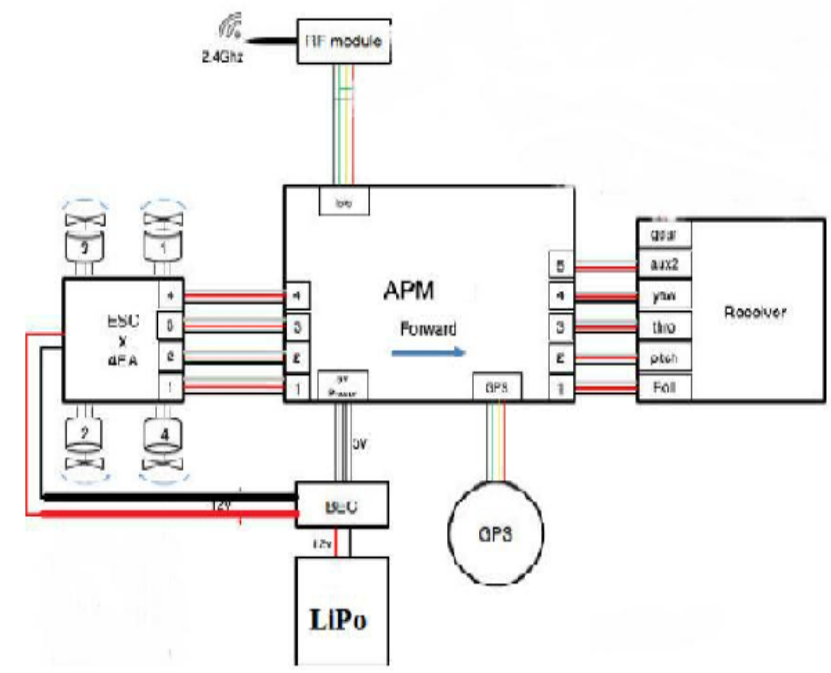

FIG - 2.1 Block Diagram

Afterward, framework was mad better to supplant thermopiles with an Inertial Measurement Unit (IMU) utilizing mix of accelerometers, gyratorsalongwithmagnetometers. Support was extended to the other vehicle types which prompted the ArduCopter, ArduPlane and ArduRoversubprojects. Earlier forms of it utilized the APM flight controller, an AVR CPU that ran the Arduino programming language that clarifies "Ardu" some portion of this venture, however nowArdupilot is as a rule $\mathrm{C}++$ which has advanced for keepingin standard the scope of equipment stages

Published By:

Blue Eyes Intelligence Engineering \& Sciences Publication

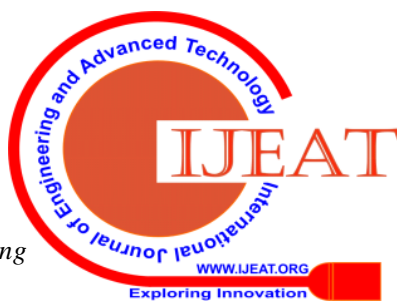


with working framework that includesPixhawk/PX4 line of autopilots, Parrot's Bebop2, Raspberry Pi based flight controllers like NAVIO2, Erle Brain and Qualcomm's Snapdragon.

Free programming methodology withinArdupilot is like that of the PX4/Pixhawk and Paparazzi Project, which has minimal effort and accessibility that empowers the specialist use in little wheeled guided air ship, for example, smaller scale air vehicles and smaller than expected UAVs.

\section{WORKING}

Drone is built and designed based on the weight and size that would provide stability, accessibility and speed during flight. Drone is controlled by ground-controlled system. Initially with the help of

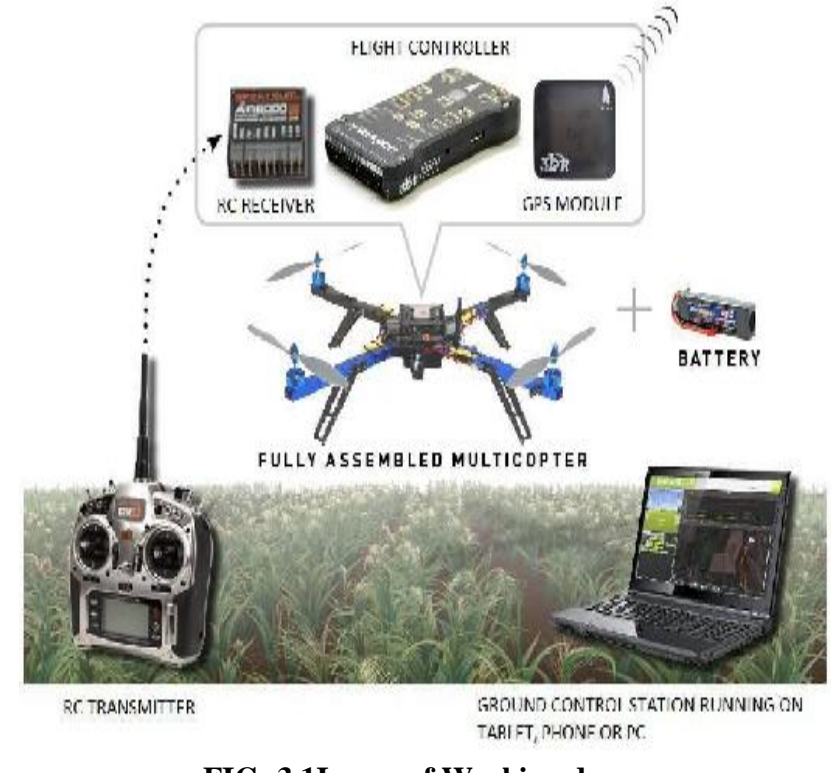

FIG -3.1Image of Working drone

e-signals to the Ardupilot Flight Controller which consists of receiver and simultaneously collects data from GPS module. Flight Controller is placed on the $\mathrm{X}$ frame on which all components are assembled. From the ground station we can operate and guide the drone.

It contains four important quadcopter controls namely Throttle, Pitch, Yaw, Roll. Roll turns your quadcopter left or right. This is done by thrusting the right stick on your transmitter to the left or vice versa. Pitch is where you push the right hand stick on the transmitter backward or forward. Yaw seems a little confusing at the start.Basically, it twists the quadcopter clockwise or counterclockwise. The propellers on the quadcopter gets enough power for getting airborne due to throttle.During the flight, you have the throttle occupiedconstantly. Forby estimating the distinction in temperature between the sky and the groundthequadcopter in the air, throttle is the control needed. Push the throttle (left stick) up very slowly, just to get the propellers going. Then stop. Repeat this again and againuntil you're comfortable with the throttle's sensitivity. Slowly push the throttleground. Then pull the throttle back down to zero and let the quadcopter land. The main general utilization of radio control frameworks in models began in the mid-1950s with single-channel self-fabricated hardware; business gear came later. The approach of transistors incredibly diminished the battery necessities, since the present prerequisites at low voltage were extraordinarily decreased and the high voltage battery was disposed of. In both cylinder and early transistor sets the model's control surfaces were typically worked by an electromagnetic escapement controlling the put away vitality in an elastic band circle, permitting basic on/off rudder control (right, left, and impartial) and now and then different capacities, for example, motor speed.
Gem controlled overly heterodyne beneficiaries with better selectivity and security made control gear increasingly able and at lower cost. Multi-channel improvements were of specific use to air ship, which truly required at least three control measurements (yaw, pitch and motor speed), rather than pontoons, which required just two or one.

As the hardware upheaval took off, single-flag channel circuit configuration ended up excess, and rather radios gave relatively coded flag streams which a servomechanism could decipher, utilizing beat position adjustment (PPM).

All the more as of late, top of the line side interest frameworks utilizing Pulse-code adjustment (PCM) highlights have gone ahead the market that give an electronic computerized bit-stream flag to the accepting gadget, rather than the prior PPM encoding type. In any case, even with this coding, loss of transmission amid flight has turned out to be progressively normal, to some extent on account of the always remote society.

Some increasingly current FM-flag collectors that still use "PPM" encoding rather can, on account of the utilization of further developed PC contributes them, be made to bolt onto and utilize the individual flag attributes of a specific PPM-type RC transmitter's outflows alone, without requiring a unique "code" transmitted alongside the control data as PCM encoding has constantly required.

In the mid-21st century, 2.4 gigahertz spread range RC control frameworks have turned out to be progressively used responsible for model vehicles and flying machine. Presently, these $2.4 \mathrm{GHz}$ frameworks are being made by most radio makers. These radio frameworks go from a few thousand dollars, right down to under US $\$ 30$ for a few.

Accordingly, alittle number of propellers use a varied pitch instrument for modifying cutting edges' pitch edge because motor speed along with air ship speed changes.

A furthermore thoughtsare the number and state of the cutting edges used. If the viewpoint proportion of cutting edges are expanded, it lessens the drag but the amount of push created depends on sharp edge region, so making use of high-perspective edges result in over the top distance of the propeller across.

Furthermore, parity is using fewer sharp edges reduces impedance that impacts in-between the edges.However,for adequate cutting edge area to transmit accessible power inside anysetwidthimplies tradeoff. Expanding number of cutting edges on a plus diminishes the amount of work all sharp edgesare required to perform, constraining nearby Mach number - a huge execution limitation on propellers.

A propeller's act undergoeswhile the cutting-edgeaccelerates near the transonic. The relative velocity inpart of a propeller is vector total of the airplane speed and distracting speed due to revolution, a propeller serrated edge tip achieves transonic speed way ahead of the air ship.

At that particular moment, while the wind streamson the tip of the edges, it achieves theminimum speed, drag and torque opposition increment immediately and the stun wave's structure makes a serrated increment in commotion. Air ship of traditiona propellers, with the lines, don't usually fly quicker than Mach 0.6.

\section{IV.THE BATTERY ELIMINATION CIRCUIT (BEC)}

The battery disposal circuit is the thing that provisions capacity to the collector and the servos. The name, battery end circuit, originates from the way that, in the "days of yore" of electric planes, you had a battery pack to control the engine and another to control the recipient. So as to spare weight, the BEC was presented 
'dispensing with' the requirement for a different beneficiary battery. A large number of the present ESC's have the BEC coordinated, making one pleasant advantageous bundle.

In the majority of our radio frameworks, the beneficiary is intended to work somewhere in the range of 4 and 6 volts. To coordinate this, the ordinary BEC supplies capacity to the beneficiary at around 5 volts by venturing down the engine battery voltage. Remember the higher the voltage of the engine battery, the harder the BEC needs to work to get the voltage down to 5 volts. As this voltage hole builds the more warmth the BEC creates.

In light of this ESC creators will confine the voltage of the BEC so watch that the BEC doesn't have an alternate voltage rating than the ESC. For instance, your ESC might be appraised for 14.8 volts however the BEC may just be evaluated for 12 volts and along these lines must be incapacitated when utilizing a battery bigger than $3 \mathrm{~s}$ (11.1 volts). For this situation you should control the collector independently.

Numerous ESCs that are planned explicitly for high voltage use don't have an incorporated BEC. BECs are appraised by how much current/amperage they can convey to therecipient. It's implied the more servos introduced in the plane, the more noteworthy the interest on the BEC. At the point when current draw on the BEC is expanded, the more warmth it creates all the while.

As indicated by Dimension Engineering, a creator of BECs, "Numerous individuals don't understand the BEC amperage rating in their ESC is deceiving. With the direct BEC incorporated with most speed controls, the amp rating diminishes as pack voltage increments. For instance, a few prevalent 25A ESCs with "3A" BECs might approve of a $2 \mathrm{~s}$ lipo pack, anyway it's solitary equipped for providing $0.5 \mathrm{~A}$ while working from a $3 \mathrm{~s}$ pack". This clearly constrains the quantity of servos that can be utilized and additionally the outstanding task at hand they can force on the BEC.

We additionally have the variable of which kind of servos are being utilized. Distinctive servos draw diverse measures of current. On the off chance that the present draw gets unreasonably high for the BEC, it will get excessively hot and cause a warm shutdown of the

\section{V.BRUSHLESS MOTOR CONTROL}

Brushless motors functions differently, and to control them is another chapter of processes. It is without brushes, that the ESC will have no idea whatsoever of the location of the coils/stator

Brushless ESC's effectivelymakes a "3 phase AC power output from a DC power source to run the motor which sends a number of AC signals generated from the ESC's circuitry. This is where the 3 wires now come in, only 2 of the wires are energized by the ESC at any one time.

The pole which is not energized at any particular instant will generate a little amount of voltage that is proportional to the speed of the motor; this is known as "Back Electromotive Force".

\section{VI.RESULT}

Advantages are simpler to appraise precisely. We have precise information on number of heart occurrences, how survival rates change when

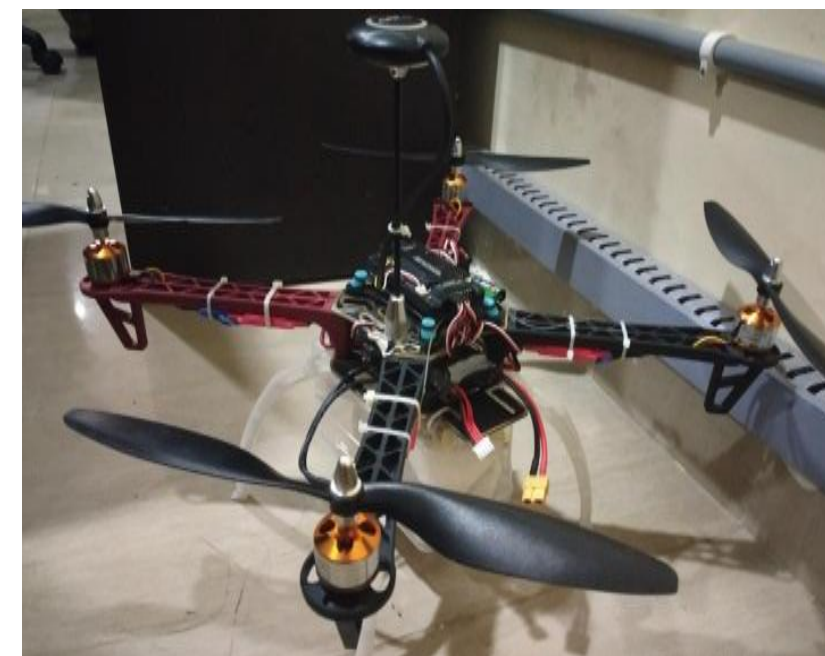

Fig - 6.1Image of constructed drone

breakdown to-stun time's change, and we can gauge lives spared by calculating in the episodes. The framework ought to react and the assessed improved reaction times. Nonetheless, progressively exact assessments of both expense and advantages are required before a helpful development can be performed.

The main minutes after a mishap are basic and fundamental to give the correct consideration to anticipate acceleration. Accelerating crisis reaction can forestall passing and quicken recuperation drastically. This is eminently valid for heart disappointment, suffocating, injuries and respiratory issues. Lifesaving innovations, for example, an Automated External Defibrillator (AED), drug, Cardiopulmonary Resuscitation (CPR) helps can be structured sufficiently reduced to be conveyed by an automaton.

\section{VII.CONCLUSION AND FUTURE SCOPE}

Heart failures guarantee a huge number of lives every year. The condition can frequently be treated with a defibrillator, yet time is a basic factor. As an outcome, survival rates are low. Ongoing improvements in automaton innovation have made regular citizen rambles modest, simple to work, and solid. This paper investigates chances to utilize Drones to convey defibrillators to out-of-medical clinic heart failure unfortunate casualties quicker than a rescue vehicle. The primary spotlight is on binding together the necessities of crisis reaction with the guidelines and guidelines required to work the Drones securely.

The examination is executed as a writing study joined with meetings. The essential partners were distinguished as crisis reaction and the Civilian Aviation Authority. The outcomes demonstrated that there was both an apparent use for emergency vehicle rambles and an approach to lawfully utilize them. The recommended methodology includes utilizing rescue vehicle rambles at games, for example, "Birkebeinerrennet" as a proof of idea, before further developed or perpetual projects are considered.

\section{REFERENCES}

1 Albulbul.A and A. Chan, "Electrode-skin impedance changes due to an externally applied force",IEEE, pp. 14, May 2012.

2 DarshanaVarma, VirendraShete, Sunil Somani,"Real Time Self Health Monitoring System", International Journal of Innovative Research in Computer and Communication Engineering (IJIRCCE), Vol. 3, Issue 6, June 2015.

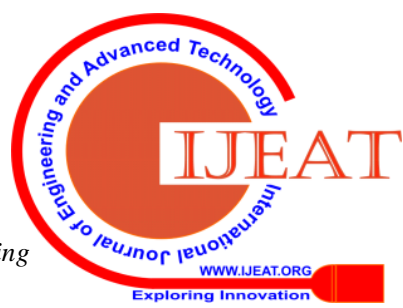


3 Farin, N., Sharif, S. and Mobin, I, “An Intelligent Sensor Based System for Real Time Heart Rate Monitoring" (HRM), Intelligent Control and Automation, 7, 55-62, May 2016.

4 Dr. FiratGüder, Dr. Alar Ainla,JuliaRedston,Prof. BobakMosadegh,Dr. Ana Glavan,T. J. Martin,Prof. George M. Whitesides,"Paper-Based Electrical Respiration Sensor" Issue 19, Pages 5727-5732, May 4, 2016.

5 JosefinLennartsson, "Strategic Placement of Ambulance Drones for Delivering Defibrillators to out of Hospital Cardiac Arrest Victims",2015.

6 Mr. Kalpesh N. Shah, Mr. Bala J. Dutt, HardikModh, "Quadrotor - An Unmanned Aerial Vehicle", International Journal of Engineering Development and Research (IJEDR), Volume 2, Issue 1, 2014.

7 Mohamed Fezari, MounirBousbia Salah, and MouldiBedda, "Microcontroller Based Heart Rate Monitor", the International Arab Journal of Information Technology, Vol. 5, No. 4, October 2008.

8 Nemati.A and M. Kumar 2014, "Modelling and control of a Single Axis Tilting Quadcopter", American Control Conference (ACC), June 4-6, 2014.

9 Shanmugasundaram.M, GMuthuselvi, SSundar, "Implementation of PIC16F877A Based Intelligent Smart Home System", International Journal of Engineering andtechnology (IJET), Vol5, Issue 2, AprMay 2013 